Original Research

\title{
The Relationship Between Hip Range of Motion and Pitching Kinematics Related to Increased Elbow Valgus Loads in Collegiate Baseball Pitchers: A Pilot Study
}

Giorgio Zeppieri Jr., PT, SCS ${ }^{1}$, Michelle L Bruner, MS, LAT, ATC ${ }^{1}$, Jon P Michelini, MS, ATC, LAT ${ }^{1}$, Kevin W Farmer, MD ${ }^{1}$

1 University of Florida Health

Keywords: baseball, elbow valgus, hip range of motion, pitching mechanics

https://doi.org/10.26603/001c.21319

\section{International Journal of Sports Physical Therapy}

Vol. 16, Issue 2, 2021

\begin{abstract}
Background
Hip range of motion (ROM) during baseball pitching is associated with trunk rotation and shoulder kinematics, which has shown to influence medial elbow valgus loading and pitching performance. The purpose of this study was to measure the relationship between hip rotational ROM and kinematic variables that influence elbow valgus loads in Division 1 collegiate pitchers.
\end{abstract}

\section{Study Design}

Descriptive laboratory study.

\section{Methods}

Three-dimensional pitching motion (Motion Analysis Corp, Santa Rosa, California) analyses were captured for seven Division 1 baseball pitchers. Six kinematic measurements related to medial elbow valgus loading were calculated while the pitchers threw fastballs. Inclinometer measurements were used to measure hip internal (IR), external (ER) ROM, and total rotational arc at the hip (IR + ER ROM). Correlations were used to evaluate the association between hip IR, ER, and total rotational arc ROM (TRARC) and six kinematic variables.

\section{Results}

Trunk angular velocity was correlated to trail hip ER and TRARC $(\mathrm{p}<0.01)$. Lead hip total arc ROM was associated with maximum shoulder ER $(\mathrm{p}<0.01)$. Lead hip IR was correlated to elbow flexion angle at ball release $(\mathrm{p}<0.01)$.

\section{Conclusion}

Hip ROM during pitching is associated with trunk angular velocity, maximum shoulder ER and elbow flexion angle at ball release. Alterations in hip TRARC appears to influence trunk rotation velocity leading to dependence on increased shoulder ROM and decreased elbow flexion angle at ball release which is associated with diminished pitching performance and excessive medial elbow valgus loads.

\section{Level of Evidence}

level 3

\footnotetext{
a Corresponding Author:

Giorgio Zeppieri Jr.

Department of Rehabilitation

University of Florida Health

3450 Hull Rd

Gainesville, FL 32608 (USA)

E-mail:zeppig@shands.ufl.edu
} 


\section{INTRODUCTION}

Increased valgus loads at the elbow joint while pitching has been shown to lead to medial elbow instability, chondral degradation at the radial head and capitellum as well as at the olecranon, and ulnar collateral ligament injury. ${ }^{1-8}$ The increased valgus loading produces tensile stresses along the medial elbow as well as compressive and shear forces along the lateral and posterior elbow. ${ }^{3,8-11}$ This development is a result of the inability of the elbow to produce sufficient varus torque to balance the accumulating valgus torque created by increasing valgus loads. ${ }^{8-11}$ Elbow valgus loading is at the most extreme when the pitching shoulder is in maximum external rotation during the late cocking and early portion of the acceleration phase. . $^{3,8-11}$

The accumulation of valgus loads can subjugate the ulnar collateral ligament (UCL) to injury either through a sudden rupture or prolonged repetitive stress. ${ }^{3,12}$ Specifically, pitchers are highly vulnerable to increased valgus loading and are at a higher risk to develop medial elbow pathology and undergo a UCL reconstruction 5.9 times greater than their non-pitching counterparts. ${ }^{13}$ Epidemiological studies demonstrated that the average rate of UCL surgeries performed in Division 1 baseball following the 2017 season occurred at rate of 0.86 surgeries per collegiate program with $85.8 \%$ of those surgical cases being pitchers. ${ }^{13}$ Additionally, the increased valgus loading will also increase the stress along the cartilaginous structures of the capitellum and the posterior-medial aspect of the olecranon leading to lateral compartment chondrosis and posterior-medial impingement during the deceleration phase. ${ }^{8,11}$

Aguinaldo et al., ${ }^{7}$ found that kinematic variables such as early trunk rotation, increased shoulder external rotation (ER), and decreased elbow flexion have been shown to increase valgus loads at the medial elbow. ${ }^{7}$ Additionally, Di Giovine et al., ${ }^{14}$ demonstrated that increased shoulder abduction during the late cocking phase resulted in increased valgus loading at the medial elbow. ${ }^{14}$ Furthermore, Werner et al., ${ }^{15}$ looked at 37 kinematic and kinetic variables and found that pitchers with increased shoulder horizontal adduction angular velocity and shoulder abduction angle at lead foot contact, elbow angle at peak valgus stress and peak shoulder external rotation torque had increased elbow valgus loading during the late cocking phase. ${ }^{15}$ In addition to these established kinematic predictors of elbow valgus loading, lower extremity range of motion (ROM) also contributes to the kinetic chain of the pitching motion. ${ }^{16-18}$

Specifically, restrictions in hip rotational ROM can influence performance and increase risk for medial elbow pathology. ${ }^{7,16,19,20}$ Restricted lead and trail hip ROM can affect the pitcher's shoulder positioning. ${ }^{21,22}$ Decreased hip ROM causes the pitching arm to be out in front of the pitcher's body forcing the pitcher to throw across their body. ${ }^{22,23}$ This scenario theoretically leads to a dampening of the transfer of energy from the lower extremities. The results lead to a potential reliance on the upper extremities to produce force needed to achieve ball velocity, leading to increased medial elbow valgus torque. ${ }^{21,22}$ Too much hip motion and the pitching arm will lag behind the already rotated trunk. ${ }^{22,23}$ In this scenario, the overhead athlete will compensate for early trunk rotation by increasing shoulder
Table 1: Descriptive Statistics for Collegiate D1 Pitchers

\begin{tabular}{|l|l|}
\hline Variable & Total Sample (N=7) \\
\hline $\begin{array}{l}\text { Age (years) } \\
\text { Mean } \pm \text { SD }\end{array}$ & $19.6 \pm 1.1$ \\
\hline $\begin{array}{l}\text { Height (meters) } \\
\text { Mean } \pm \text { SD }\end{array}$ & $1.89 \pm 0.06$ \\
\hline $\begin{array}{l}\text { Weight (Kilograms) } \\
\text { Mean } \pm \text { SD }\end{array}$ & $93.3 \pm 6.8$ \\
\hline Throwing Hand Dominance & Right handed $=5$ \\
& Left handed $=2$ \\
\hline College Playing Year & Junior $=2$ \\
\hline $\begin{array}{l}\text { Ball velocity (miles per hour) } \\
\text { Mean } \pm \text { SD }\end{array}$ & Sophomore $=3$ \\
\hline
\end{tabular}

*Standard Deviation

ER ROM. Increased shoulder ER ROM has been associated with increased valgus loads at the medial elbow. 7,21,22

Given the interaction between hip motion and pitching mechanics, a salient link may exist between restrictions in hip rotational ROM and medial elbow dysfunction. Thus, the purpose of this exploratory study was to measure the relationship between hip rotational ROM and kinematic variables that influence elbow valgus loads in Division 1 collegiate pitchers. Determining if a relationship exists between hip ROM and valgus forces at the elbow may aid in developing interventions to address poor pitching mechanics, while improving pitching performance.

\section{MATERIALS AND METHODS}

\section{PARTICIPANTS}

A total of seven Division 1 collegiate baseball pitchers (mean age, $19.57 \pm 1.1$ years; mean height, $1.89 \pm .6 \mathrm{~cm}$; mean weight, $93.3 \pm 6.8 \mathrm{~kg}$ ) consented to participate in this study. (Table 1) Five subjects were right-handed dominant and two subjects were left-handed dominant. All subjects were healthy at the time of testing, currently active, reported no trunk, upper or lower extremity injury in the past six months or had history of upper or lower extremity surgery. Prior to participation, all subjects signed a written informed consent approved by the University of Florida Health Science Center Institutional Review Board (IRB-01).

\section{PROCEDURES}

All subjects were tested prior to the beginning of preseason workouts. All hip rotational ROM measures were performed by the same two American Physical Therapy Association (APTA) board sports certified specialist physical therapists. One to stabilize and one to record measurements. Hip ROM testing occurred prior to kinematic testing at the University 
Table 2: Hip Range of Motion and Kinematic Variables Measurements for Collegiate D1 Baseball Pitchers

\begin{tabular}{|l|l|}
\hline Measurements & Mean \pm SD \\
\hline Trail Hip ER ROM (degrees) & $34.3 \pm 7.5^{\circ}$ \\
\hline Lead Hip ER ROM (degrees) & $29.4 \pm 8.9^{\circ}$ \\
\hline Trail Hip IR ROM (degrees) & $12.6 \pm 3.6^{\circ}$ \\
\hline Lead Hip IR ROM (degrees) & $16.9 \pm 8.5^{\circ}$ \\
\hline Trail Hip TRARC (degrees) & $46.9 \pm 5.6^{\circ}$ \\
\hline Lead Hip TRARC (degrees) & $46.3 \pm 11.5^{\circ}$ \\
\hline Maximum Shoulder External Rotation (degree) & $171.6 \pm 8.4^{\circ}$ \\
\hline Shoulder Abduction Angle at Foot Contact (degree) & $91.6 \pm 9.5^{\circ}$ \\
\hline Elbow Flexion Angle at Ball Release (degree) & $21.87 \pm 5.5^{\circ}$ \\
\hline Maximum Shoulder Horizontal Adduction Angular Velocity (degree/second) & $1315.7 \pm 257.4^{\circ} / \mathrm{s}$ \\
\hline Onset of Maximum Trunk Angular Velocity (\% of Pitching Cycle) & $95 \pm 6.2 \%$ \\
\hline Onset of Maximum Elbow Flexion (\% of Pitching Cycle) & $105.9 \pm 1.9 \%$ \\
\hline
\end{tabular}

$\mathrm{ER}=$ External rotation, $\mathrm{ROM}=$ range of motion, $\mathrm{IR}=$ Internal rotation, $\mathrm{TRARC}=$ total rotational arc range of motion

of Florida Human Dynamics Laboratory.

\section{HIP ROTATIONAL ROM MEASUREMENTS}

Hip ER and internal rotation (IR) ROM measurements were taken with a bubble inclinometer while the subject was placed in a prone position with hip of interest in 0 degrees of extension and abduction with knee in 90 degrees of flexion. ${ }^{24,25} \mathrm{~A}$ two-examiner method was utilized. One examiner placed one hand on the testing hip greater trochanter and pelvis to minimize excess movement and used the other hand to grip the subject lower leg to passively move the hip until first resistance was detected. The second examiner placed the bubble inclinometer proximal to the medial malleolus aligned with the shaft of the tibia to record ER and IR ROM. Total hip rotational ROM was calculated as the sum of hip ER and IR ROM. This measuring method has shown good interclass correlation $(\mathrm{ICC}=.98) .{ }^{24}$

\section{PITCH BIOMECHANICS PREPARATION}

Motion capture set-up was based on previously validated methods. ${ }^{26,27}$ Motion analysis was captured with a highspeed, 12-camera optical motion capture system (Motion Analysis Corp, Santa Rosa, California). Data were captured at $200 \mathrm{~Hz}$. Fifteen reflective markers were applied bilaterally at the lateral tip of the acromion, lateral humeral epicondyle, greater trochanter, lateral femoral epicondyle, lateral malleolus, and hallux; additional markers were placed on the radial and ulnar styloid processes of the dominant hand with one marker placed on the non-dominant radioulnar joint. ${ }^{26,28}$ Reflective tape was placed on a standard NCAA collegiate ball.

Each player was instructed warm-up based on their standard team regulated stretching and throwing preparation routine. The players received standardized instructions to pitch ten game-effort pitches from the wind-up position off an indoor mound (with a standard slope of one inch in height for every foot nearer to home plate); all pitches were 2-seam fastballs. Each player pitched into a marked area along a wall net that was the same size as home plate (43.18 $\mathrm{cm}$ X $43.18 \mathrm{~cm}$ ). Data was collected from the three pitches with the greatest accuracy and velocity. Ball velocity was measured with a radar gun.

The pitching cycle (lead foot contact to ball release) was normalized to $100 \% .^{26,29}$ The variables calculated included: 1) three joint angles (maximum shoulder ER during the pitching cycle, shoulder abduction angle at foot contact, and elbow flexion angle at ball release); 2) one angular velocity (maximum shoulder horizontal adduction angular velocity); and 3) two variables (initiation of maximum trunk angular velocity and initiation of maximum elbow flexion). These variables were calculated as the percentage of the pitching cycle at which the event occurred. These variables were chosen based on their influence on elbow valgus loading during the cocking and acceleration phase. $7,14,15,22,30$

\section{STATISTICAL ANALYSIS}

All statistical analyses were performed with SPSS (version 22.0; IBM Corp., Chicago, IL, USA). Descriptive statistics (mean and standard deviation) were calculated for Hip ROM and kinematic variable measurements. (Table 2) Pearson's product correlations were used to evaluate the association between lead and trail hip IR, ER, and total rotational ROM and the six kinematic variables. Correlations were deemed significant if $\mathrm{p}$ was less than 0.01 .

\section{RESULTS}

The correlations between Hip ROM and biomechanical variables are shown in Table 3.

\section{RELATIONSHIP BETWEEN TRAIL HIP ROM AND BIOMECHANICAL VARIABLES}

The trail hip TRARC was significantly related to the onset 
Table 3: The Relationship Between Hip Range of Motion and Biomechanical Variables for Collegiate D1 Baseball Pitchers

\begin{tabular}{|l|l|l|l|l|l|l|}
\hline \multicolumn{7}{|l|}{ Hip Range of Motion } \\
\hline
\end{tabular}

* Statistically significant correlation $(\mathrm{p}<.01)$

of trunk angular velocity $(\mathrm{r}=.712, \mathrm{p}=.003)$. There was also a significant correlation between trail hip ER and the initiation of trunk angular velocity $(r=.650, p=.009)$. (Figures $\underline{1 \mathrm{~A}}$ and $\underline{1 \mathrm{~B}})$

\section{RELATIONSHIP BETWEEN LEAD HIP ROM AND BIOMECHANICAL VARIABLES}

The lead hip total arc of motion was significantly related to maximum shoulder ER $(r=695, \mathrm{p}=.005)$. (Figure $1 \mathrm{C})$ There was a significant correlation between elbow flexion angle at ball release and lead hip IR $(r=.952, p=.001)$. (Figure 1D)

\section{DISCUSSION}

The influence of hip ROM is vital to developing obligatory trunk and upper extremity torque and ball velocity during the pitching motion. ${ }^{19-21,31,32}$ Most collegiate pitchers will either generate power through their stance leg in a load and drive strategy or by converting the force generated by their body moving towards their intended target into ball velocity by rotating around their stride leg. ${ }^{8,19,31,32}$ A decrease in hip ROM may initiate a series of compensatory actions within the pitching motion which may lead to increased elbow valgus torque and loading. 2,7,10,14,16,19,22,32 The current findings suggest that there may be an association between hip ROM and established kinematic influencers of increased elbow valgus torque during the pitching motion.

Lee et al. ${ }^{33}$ described the hip as being the principle structure initiating the spine to rotate. ${ }^{33}$ From a kinematic perspective, the trail hip will initiate pelvic rotation which will be followed by trunk rotation which will influence lead foot contact position and overall pelvic orientation. If there is too much hip rotation, then the lead foot and pelvis will be in a more open position. ${ }^{21-23,34}$ Conversely, if there were too little hip rotation, the lead foot and pelvis would be

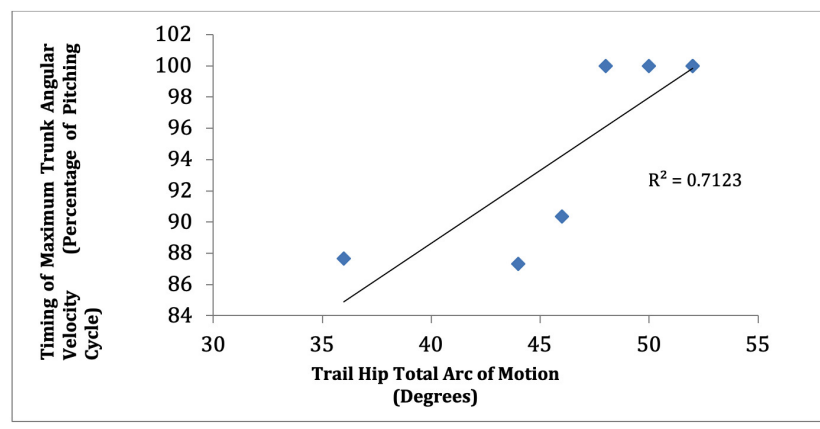

Figure 1A: Relationship Between Time of Onset of Maximum Trunk Velocity and Trail Hip Total Arc of Motion

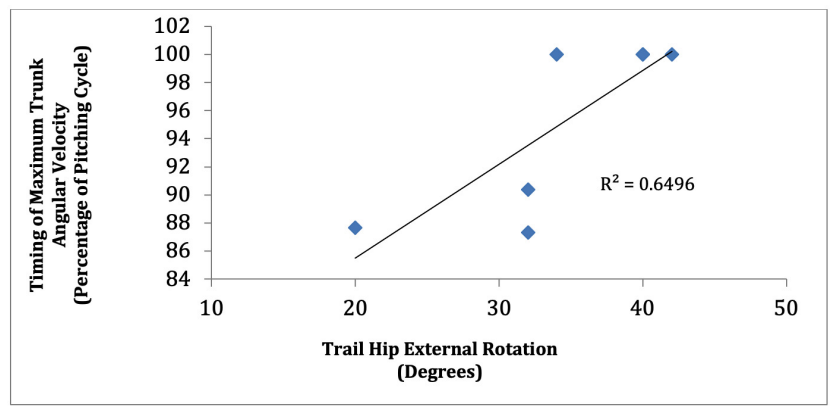

Figure 1B: Relationship Between Time of Onset of Maximum Trunk Velocity and Trail Hip External Rotation

in a more closed position. ${ }^{21-23,33}$ Fortenbaugh et al., ${ }^{35} \mathrm{de}-$ scribed that improper timing of pelvic rotation and trunk 
rotation would decrease pitching performance and increase stresses on the anterior shoulder and medial elbow. ${ }^{35}$ The authors reported that pelvic rotation should occur between $28 \%$ and $35 \%$ of the pitching cycle while trunk rotation should occur between $47 \%$ and $53 \%$ of the pitching cycle. 35 The current findings demonstrated a correlation between trail hip total arc of motion and trunk angular velocity, which may provide further support showing that hip rotational ROM is correlated to pelvic orientation and trunk rotation and velocity. Additionally, decreased trail hip ER will restrict the forward movement of the pitcher's trunk over the lead leg dampening the transfer of energy from the lower extremities leading to increased shoulder ER. However, the current findings did not demonstrate a correlation between lead hip rotational ROM and trunk angular velocity. We believe that our findings were due to our small sample size and although the results did not reach statistical significance, they did demonstrate that pitchers who rotated their trunks earlier in the pitching cycle appeared to have increased lead hip total arc of motion.

The current findings demonstrate that there is an association between lead hip total arc of rotational motion and maximal shoulder ER. This finding is consistent with previously reported findings showing that diminished as well as excessive lead hip rotational ROM can influence throwing shoulder ER during the late cocking and early acceleration phase. ${ }^{7,36}$ For example, inadequate lead hip ROM can cause a domino effect leading to decreased stride length and damped lower extremity force production. ${ }^{16,21}$ The pitcher will be forced to throw across his body to compensate for the lack of force production by increasing shoulder ER to generate ball velocity. ${ }^{19}$ As a result, the pitcher may rely on shoulder rotation as the primary force generator while pitching. This method of force generation relies on increasing shoulder ER to rapidly move into IR to generate ball velocity, which has be shown to increase shoulder ER and elbow valgus torque, which may not only have a detrimental effect on pitching performance, but may lead to medial elbow pathology. 7,8,10,22 Increased lead hip ROM will lead to the opposite effect, where the pitching shoulder lags behind the early rotating trunk. ${ }^{22,23}$ In this scenario, the pitcher will also have to compensate for reduced ball velocity by increasing shoulder ER. In this instance however, due early pelvic rotation, the pitcher has to compensate by speeding up the pitching arm to compensate for the de-rotated trunk. Again, due to early trunk rotation, the shoulder rapidly rotating from ER to IR would generate ball velocity. In both instances, shoulder ER is increased which may lead to increased medial elbow tensile stresses. $7,8,22$

The current data also showed that lead hip IR correlated to elbow flexion angle at ball release. Aguinaldo et al., ${ }^{7}$ demonstrated that a decrease in elbow flexion angle was linked to an increase in elbow valgus torque. ${ }^{7}$ The authors surmised that this occurred because increased elbow flexion would reduce the lever arm (throwing arm) around the trunk as the pitcher moves toward the intended target. ${ }^{7}$ The shorter lever arm would lead to decreased elbow valgus torques compared to a more extended elbow, which would act as a longer lever. ${ }^{7}$ A more extended elbow during the pitching motion may be indicative of a throwing shoulder that lags behind a rotating trunk. ${ }^{7}$ This shoulder lag can be

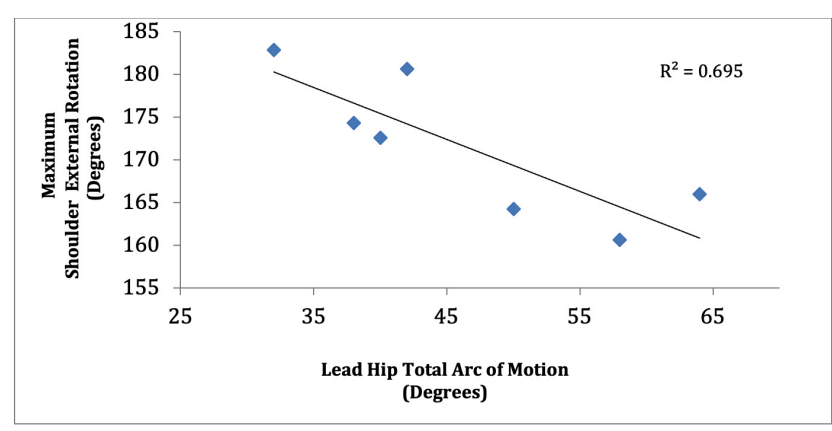

Figure 1C: Relationship Between Maximum Shoulder External Rotation and Lead Hip Total Arc of Motion ROM

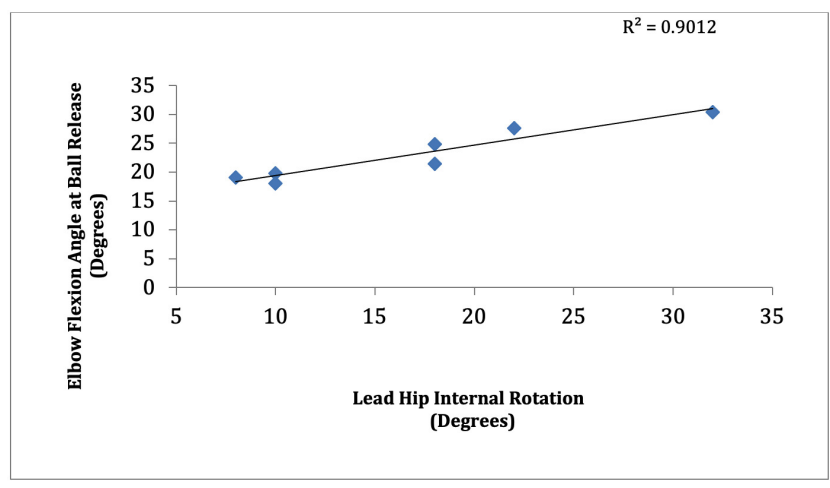

Figure 1D: Relationship Between Elbow Angle at Ball Release and Lead Hip Internal Rotation

linked to excessive lead hip IR. However, the findings of the current study demonstrated an average lead hip IR of 16.9 degrees, which is a smaller angle that previous research has shown to be ideal for pitchers. ${ }^{35,37}$ Such findings would suggest restricted lead hip IR in this cohort. Sekiguchi et al., ${ }^{38}$ found that decreased lead hip IR was significantly associated with an increased risk for shoulder and elbow pathology in a 9-12 year pitching cohort. ${ }^{38}$ Although younger than the current cohort, the authors believe that a similar situation may occur in the studied population, where decreased lead hip IR would lead to early pelvic rotation speeding up ball delivery and therefore altering the timing of elbow flexion at foot contact and may increase valgus loading along the elbow.

The current findings did not demonstrate a correlation between lead and trail hip ROM and maximum shoulder horizontal adduction angular velocity and shoulder abduction angle at foot contact. Previous published research has demonstrated that pitchers with excessive horizontal adduction during late cocking in a situation where the pitcher leads with his elbow will increase medial elbow stress due increased varus elbow torque. ${ }^{35,39-41}$ In addition Matsuo et al., ${ }^{42}$ showed that at late cocking pitchers with an angle less than 90-100 degrees of abduction during foot contact would demonstrate increased elbow varus torque. ${ }^{35,42}$ Both scenarios, a pitcher leading with his elbow and or with decreased shoulder abduction would increase deleterious 
stresses along their medial elbow and diminished ball velocity.

The results can be used to gain preliminary insight to the relationship between hip ROM and pitching kinematics related to elbow valgus loading. Although it may be difficult to address and measure pitching kinematics or kinetics within the clinic, a thorough understanding of hip anatomy, pathomechanics, and muscle physiology can assist with making informed decisions regarding the genesis of injury when evaluating the pitcher. While, the current study was not powered to determine injury risk, there seems to be a relationship between hip ROM as a clinically modifiable factor and pitching kinematics related to elbow valgus loading. ${ }^{43,44}$ These findings suggest hip ROM should be examined as part of the overhead athlete assessment during the evaluation process.

This study has several limitations when interpreting the results. The sample size is limited in number and consisted of seven Division 1 baseball pitchers, which may have been too small to accurately assess relationships. However, collegiate pitching populations are historically small and accessibility to this population and measurements within a lab can be quite difficult to ascertain. Additionally, the results cannot be extrapolated to other age (youth) or level (professionals). Furthermore, intra-rater reliability was not assessed when measuring hip ROM in the prone position in this study. However, reliability has been previously reported for the hip ROM measurements positioning and technique that we incorporated. The authors utilized the same two clinical examiners in the same role, one to stabilize and one measure for every athlete. ${ }^{24,25}$ Kinematic variables were acquired in a laboratory using an artificial mound, which is not identical to pitching on the field.

\section{CONCLUSION}

In conclusion, previous literature has described the relationship between specific kinematic and kinetic variables and medial elbow valgus loading. $7,8,10,22,36,39,42$ The findings of the current study elucidate the relationship between hip ROM and specific kinematic predictors of medial elbow valgus loading; i.e. initiation of trunk rotation, increased maximum ER, and increased elbow flexion angle at ball release. Understanding the relationship between hip ROM and pitching kinematics related to increased medial elbow valgus loading is instrumental for healthcare and performance providers to understand how alterations hip ROM may affect the pitching motion and consequentially predispose the pitcher to injury and decreased performance. Additionally, hip ROM should be screened by practitioners when determining prevention and rehabilitation programs for collegiate pitchers.

\section{DISCLOSURES}

This study was approved by the University of Florida Health Science Center Institutional Review Board (IRB-01). The authors certify that they have no affiliations with or financial involvement in any organization or entity with a direct financial interest in the subject matter or materials discussed in the article.

Submitted: May 17, 2020 CDT, Accepted: October 10, 2020 CDT 


\section{REFERENCES}

1. Erickson BJ, Chalmers PN, Axe MJ, Romeo AA. Exceeding pitch count recommendations in Little League baseball increases the chance of requiring Tommy John surgery as a professional baseball pitcher. Orthop J Sport Med.

2017;5(3):2325967117695085. doi:10.1177/232596711 $\underline{7695085}$

2. Flesig GS, Andrews JR, Dillman CJ, Escamilla RF. Kinetics of baseball pitching with implications about injury mechanisms. Am J Sports Med. 1995;23(2):233-239. doi:10.1177/03635465950230021 $\underline{8}$

3. DeFroda SF, Goodman AD, Gil JA, Owens BD. Epidemiology of elbow ulnar collateral ligament injuries among baseball players: National collegiate athletic association injury surveillance program, 2009-2010 through 2013-2014. Am J Sports Med. 2018;46(9):2142-2147. doi:10.1177/036354651877331 $\underline{4}$

4. Chen FS, Rokito AS, Jobe FW. Medial elbow problems in the overhead-throwing athlete. J Am Acad Orthop Surg. 2001;9(2):99-113. doi:10.5435/0012 4635-200103000-00004

5. Hang Y-S, Lippert FG III, Spolek GA, Frankel VH, Harrington RM. Biomechanical study of the pitching elbow. Int Orthop. 1979;3(3):217-223. doi:10.1007/bf0 $\underline{0265715}$

6. Wilson FD, Andrews JR, Blackburn TA, Mccluskey $\mathrm{G}$. Valgus extension overload in the pitching elbow. Am J Sports Med. 1983;11(2):83-88. doi:10.1177/03635 $\underline{4658301100206}$

7. Aguinaldo AL, Chambers H. Correlation of throwing mechanics with elbow valgus load in adult baseball pitchers. Am J Sports Med. 2009;37(10):2043-2048. do i:10.1177/0363546509336721

8. Anz AW, Bushnell BD, Griffin LP, Noonan TJ, Torry MR, Hawkins RJ. Correlation of torque and elbow injury in professional baseball pitchers. Am J Sports Med. 2010;38(7):1368-1374. doi:10.1177/0363546510 $\underline{363402}$

9. Conte SA, Flesig GS, Dines JS, et al. Prevalence of ulnar collateral ligament surgery in professional baseball players. Am J Sports Med. 2015;43(7):1764-1769. doi:10.1177/036354651558079 2
10. Werner SL, Murray TA, Hawkins RJ, Gill TJ. Relationship between throwing mechanics and elbow valgus in professional baseball pitchers. J Shoulder Elbow Surg. 2002;11(2):151-155. doi:10.1067/mse.200 $\underline{2.121481}$

11. Dugas JR. Valgus extension overload: Diagnosis and treatment. Clin Sports Med. 2010;29(4):645-654. $\underline{\mathrm{d}}$ oi:10.1016/j.csm.2010.07.001

12. Grace SP, Field LD. Chronic medial elbow instability. Orthop Clin North Am. 2008;39(2):213-219. doi:10.1016/j.ocl.2007.12.003

13. Rothermich MA, Conte SA, Aune KT, Fleisig GS, Cain ELJ, Dugas JR. Incidence of elbow ulnar collateral ligament surgery in collegiate baseball players. Orthop J Sport Med. 2018;6(4):2325967118764657. doi:10.1177/232596711 $\underline{8764657}$

14. DiGiovine NM, Jobe FW, Pink M, Perry J. An electromyographic analysis of the upper extremity in pitching. J Shoulder Elbow Surg. 1992;1(1):15-25. doi:1 0.1016/s1058-2746(09)80011-6

15. Werner SL, Murray TA, Hawkins RJ, Gill TJ. Relationship between throwing mechanics and elbow valgus in professional baseball pitchers. J Shoulder Elbow Surg. 2002;11(2):151-155. doi:10.1067/mse.200 2.121481

16. Laudner KG, Moore SD, Sipes RC, Meister K. Functional hip characteristics of baseball pitchers and position players. Am J Sports Med. 2010;38(2):383-387. doi:10.1177/0363546509347365

17. Anloague PA, Spees V, Smith J, Herbenick MA, Rubino LJ. Glenohumeral range of motion and lower extremity flexibility in collegiate - level baseball players. Sports Health. 2012;4(1):25-30. doi:10.1177/1 $\underline{941738111422336}$

18. Fleisig GS, Escamilla RF, Andrews JR. Biomechanics of throwing. In: Zachazewski JE, Magee DJ, Quillen WS, eds. Athletic Injuries and Rehabilitation. Philadelphia, PA: WB Saunders; 1996:332-353.

19. Sauers EL, Huxel Bliven KC, Johnson MP, Falsone $\mathrm{S}$, Walters S. Hip and glenohumeral rotational range of motion in healthy professional baseball pitchers and position players. Am J Sports Med. 2013;42(2):430-436. doi:10.1177/0363546513508537 
20. Crawford EA, Whiteside D, Denewerth JM, Ross JR, Bedi A, Goulet GC. In vivo hip morphology and kinematics in elite baseball pitchers. Arthroscopy. 2016;32(5):798-805. doi:10.1016/j.arthro.2015.11.052

21. Saito M, Kenmoku T, Kameyama K, et al. Relationship between tightness of the hip joint and elbow pain in adolescent baseball players. Orthop J Sports Med. 2014;2(5):2325967114532424. doi:10.117 $\underline{7 / 2325967114532424}$

22. Calabrese GJ. Pitching mechanics, revisited. Int J Sports Phys Ther. 2013;8(5):652-660.

23. Wilk KE, Meister K, Flesig G, Andrews JR. Biomechanics of the overhead throwing motion. Sports Med Arthrosc Rev. 2000;8(2):124-134. doi:10.10 97/00132585-200008020-00002

24. Ellenbecker TS, Ellenbecker GA, Roetert EP, Silva RT, Keuter G, Sperling F. Descriptive profile of hip rotation range of motion in elite tennis players and professional baseball players. Am J Sports Med. 2007;35(8):1371-1376. doi:10.1177/036354650730026 $\underline{0}$

25. Zeppieri G Jr, Lentz TA, Moser MW. Changes in hip range of motion and strength in collegiate baseball pitchers over the course of a competative season: A pilot study. Int J Sports Phys Ther. 2015;10(4):505-13:505-513.

26. Flesig GS, Barrentine SW, Zheng N, Escamilla RF, Andrews JR. Kinematic and kinetic comparison of baseball pitching among various levels of development. J Biomechanics. 1999;32(12):1371-1375. doi:10.1016/s0021-9290(99)00127-x

27. Wasser JG, Chen C, Vincent HK. Kinematics of shooting in high school and collegiate lacrosse players with and without low back pain. Orthop J Sports Med. 2016;4(7):2325967116657535. doi:10.117 $\underline{7 / 2325967116657535}$

28. Vincent HK, Chen C, Zdziarski LA, Montes J, Vincent KR. Shooting motion in high school, collegiate, and professional men's lacrosse players. Sports Biomech. 2015;14(4):448-458. doi:10.1080/147 63141.2015.1084034

29. Fleisig GS, Escamilla RF, Andrews JR, Matsuo T, Satterwhite Y, Barrentine SW. Kinematic and kinetic comparison between pitching and football passing. $J$ Applied Biomech. 1996;12(2):207-224. doi:10.1123/ja b.12.2.207

30. Toyoshima S, Hoshikawa T, Miyashita M, Oguri T. Contribution of the body parts to throwing performance. In: Biomechanics IV. Baltimore: University Park press; 1974:169-174. https://doi.org/1 0.1007/978-1-349-02612-8 24.
31. MacWilliams BA, Choi T, Perezous MK, Chao EYS, McFarland EG. Characteristic ground-reaction forces in baseball pitching. Am J Sports Med. 1998;26(1):66-71. doi:10.1177/0363546598026001280 1

32. Kibler WB. Pathophysiology of overload injuries around the elbow. Clin Sports Med. 1995;14(2):447-457. doi:10.1016/s0278-5919(20)3023 $\underline{5-0}$

33. Lee RYW, Wong TKT. Relationship between the movements of the lumbar spine and hip. Hum Mov Sci. 2002;21(4):481-494. doi:10.1016/s0167-9457(02)0 $\underline{0117-3}$

34. Robb AJ, Flesig G, Wilk K, Macrina L, Bolt B, Pajaczkowski J. Passive range of motion of the hips and their relationship with pitching biomechanics and ball velocity in professional baseball pitchers. Am J Sports Med. 2010;38(12):2487-2493. doi:10.1177/036 $\underline{3546510375535}$

35. Fortenbaugh D, Flesig GS, Andrews JR. Baseball pitching biomechanics in relation to injury risk and performance. Sports Health. 2009;1(4):314-320. doi:1 $\underline{0.1177 / 1941738109338546}$

36. Laudner K, Wong R, Onuki T, Lynall R, Meister K. The relationship between clinically measured hip rotational motion and shoulder biomechanics during the pitching motion. J Sci Med Sport.

2015;18(5):581-584. doi:10.1016/j.jsams.2014.07.011

37. Fleisig GS, Kingsley DS, Loftice JW, et al. Kinetic comparison among the fastball, curveball, change-up, and slider. Am J Sports Med. 2006;34(3):423-430. doi:1 $0.1177 / 0363546505280431$

38. Sekiguchi T, Hagiwara Y, Yabe Y, et al. Restriction in the hip internal rotation of the stride leg is associated with elbow and shoulder pain in elite young baseball players. J Shoulder Elbow Surg. 2020;29(1):139-145. doi:10.1016/j.jse.2019.07.004

39. Andrews JR, Heggland EJH, Flesig GS, Zheng N. Relationship of ulnar collateral ligament strain to amount of medial olecranon osteotomy. Am J Sports Med. 2001;29(6):716-721. doi:10.1177/036354650102 90060801

40. Kaminenu S, El Attrache NS, O'Driscoll SW, et al. Medial collateral ligament strain with partial posterior olecranon resection: a biomechanical study. J Bone Joint Surg Am. 2004;86(11):2424-2430. doi:10.2 $\underline{106 / 00004623-200411000-00010}$

41. Levin JS, Zheng N, Dugas J, Cain EL, Andrews JR. Posterior olecranon resection and ulnar collateral ligament strain. J Shoulder Elbow Surg. 2004;13(1):66-71. doi:10.1016/i.jse.2003.09.010 
42. Matsuo T, Escamilla RF, Flesig GS, Barrentine SW, Andrews JR. Comparison of kinematic and temporal parameters between different pitch velocity groups. $J$ Appl Biomech. 2001;17(1):1-13. doi:10.1123/jab.17.1.1
44. Chalmers PN, Wimmer MA, Verma NN, et al. The relationship between pitching mechanics and injury: A review of current concepts. Sports Health. 2017;9(3):216-221. doi:10.1177/1941738116686545

43. Escamilla RF, Andrews JR. Shoulder muscle recruitment patterns and related biomechanics during upper extremity sports. Sports Med. 2009;39(7):569-590. doi:10.2165/00007256-20093907 $\underline{0-00004}$ 